\section{$\underset{\text { hommes }}{\text { \& migrations }}$}

\section{Hommes \& migrations}

Revue française de référence sur les dynamiques

migratoires

$1313 \mid 2016$

1983, le tournant médiatique

\title{
Les enfants de l'immigration au Centre Pompidou
}

Espoirs et malentendus de la mise en scène des cultures immigrées dans la France des années 1980

\section{Adèle Momméja}

\section{(2) OpenEdition}

\section{Journals}

Édition électronique

URL : http://journals.openedition.org/hommesmigrations/3569

DOI : 10.4000/hommesmigrations.3569

ISSN : 2262-3353

Éditeur

Musée national de l'histoire de l'immigration

Édition imprimée

Date de publication : 1 janvier 2016

Pagination : $97-102$

ISBN : 978-2-919040-34-6

ISSN : 1142-852X

Référence électronique

Adèle Momméja, "Les enfants de l'immigration au Centre Pompidou », Hommes \& migrations [En

ligne], 1313 | 2016, mis en ligne le 01 janvier 2019, consulté le 17 mars 2020. URL : http://

journals.openedition.org/hommesmigrations/3569; DOI : https://doi.org/10.4000/

hommesmigrations.3569 


\title{
LES ENFANTS DE L'IMMIGRATION AU CENTRE POMPIDOU ESPOIRS ET MALENTENDUS DE LA MISE EN SCĖNE DES CULTURES IMMIGRÉES DANS LA FRANCE DES ANNÉES 1980
}

par ADĖLE MOMMÉJA, doctorante au laboratoire Sophiapol, université Paris-Ouest Nanterre La Défense

\author{
L'exposition Les enfants de l'immigration qui s'est tenue \\ au Centre George Pompidou entre janvier et avril 1984 \\ avait comme objectif d'ouvrir une scène nationale aux créations \\ artistiques d'un groupe social marginalisé. Cette exposition \\ a offert une visibilité importante à des artistes qui interrogent, \\ dans leurs œuvres, leur rapport à l'immigration. Les non-dits \\ et les ambiguïtés du processus de sélection des exposants \\ instillent néanmoins un doute sur les véritables raisons \\ de la présence de ces artistes « d'origine immigrée » dans \\ ce haut lieu de l'art contemporain.
}

Le 8 janvier 1984 Jack Lang et Georgina Dufoix, respectivement ministre de la Culture et secrétaire d'État à la Famille, à la Population et aux Travailleurs immigrés, inaugurent l'exposition Les enfants de l'immigration qui se tient au Centre Pompidou jusqu'au 23 avril 1984. L'exposition présente des œuvres plastiques, des vidéos, des photographies et des sculptures qui témoignent de la vie quotidienne des " enfants d'immigrés » et des problèmes auxquels ils sont confrontés, tels que le racisme, le chômage et la vie dans les quartiers relégués. L'exposition comporte également une partie "spectacle vivant » où sont présentés des pièces de théâtres et des débats politiques sur le « vote beur » ou la « culture beur » avec des interventions de représentants des institutions. Quelques mois avant l'ouverture, le projet menaçait pourtant de péricliter en raison de difficultés financières. L'intervention de Pierre Saragoussi, membre de la Caisse des dépôts et consignations, auprès du Premier ministre Pierre Mauroy, permet d'obtenir des financements du ministère de la Culture et du Fonds d'action sociale (FAS) et de mener l'exposition à son terme. Cette inauguration en grande pompe, le soutien financier apporté par l'État et les nombreux articles que la presse nationale a consacrés à l'exposition témoignent de l'intérêt que suscite ce projet. Cet intérêt ne semble 
pas surprenant dans la mesure où le titre de l'exposition renvoie explicitement à un groupe social, les " enfants de l'immigration ", qui fait l'objet d'une visibilité croissante depuis les émeutes de Lyon et la Marche pour l'égalité et contre le racisme.

Si les acteurs du débat public ne s'entendent pas sur les solutions qu'il conviendrait d'apporter au " problème " des " enfants d'immigrés », il existe peu de voix pour contester l'existence d'un tel problème. En l'intitulant Les enfants de l'immigration, les concepteurs de l'exposition posent leur pierre à l'édifice de "l'invention » de ce nouveau problème social. Ce texte propose de reconstituer le travail des différents acteurs qui ont participé à la préparation de l'exposition ${ }^{1}$. L'analyse des dilemmes auxquels ils ont été confrontés et des choix qu'ils ont faits au moment de sélectionner les artistes est significative du rapport que les représentants des institutions entretiennent avec ce groupe des " enfants de l'immigration " que l'exposition met à l'honneur.

\section{Des commissaires d'exposition à la rencontre des enfants d'immigrés}

Les premiers documents qui présentent l'exposition indiquent qu'un de ses objectifs principaux est d'ouvrir les portes de cette institution de l'art contemporain à un groupe marginalisé pour permettre son insertion dans la société française. Il s'agit d'ouvrir une scène aux "enfants d'immigrés » afin qu'ils témoignent de leurs conditions de vie et des problèmes qu'ils rencontrent au quotidien. Les commissaires de l'exposition insistent, dans les entretiens, sur l'état d'esprit d'« ouverture » qui les animait alors. Les scénographes conçoivent ainsi un espace ouvert où le public peut facilement circuler d'un espace à l'autre. De même, la partie « spectacle vivant » entend briser l'aspect solennel et austère du lieu afin d'attirer des publics peu habitués à fréquenter les musées. L'exposition est aussi conçue comme une entreprise de sensibilisation de l'opinion publique à « la réalité du pluralisme ethnique et culturel de la société française ${ }^{2}$ ». Le thème de la « rencontre » entre les « enfants d'immigrés » et la société française est régulièrement mobilisé pour expliquer le projet de l'exposition : «Permettre que ces expressions, le plus souvent cantonnées aux milieux de l'immigration, soient accessibles à un large public ; témoigner de manière vivante d'une interculturalité déjà en acte dans cette génération, tels sont les buts de cette manifestation ${ }^{3}$."

Interrogés sur les raisons de cet investissement personnel et professionnel, les acteurs évoquent le contexte politique de l'époque et l'immense enthousiasme qu'a suscité l'arrivée de la gauche au pouvoir. Ils se souviennent d'une époque utopique marquée par l'espoir de transformer la société : «On n'a vraiment pas eu de difficultés. Nous étions dans un dynamisme très fort à l'époque. Il faut se souvenir de la période, tout était possible en 1983 en terme d'ouverture politique, culturelle ${ }^{4}$. » Ce souvenir d'un soudain élargissement des possibles contraste avec les analyses historiques qui associent l’année 1983 à la conversion des élites socialistes à la rigueur.

Dans le domaine des politiques économiques, les dirigeants socialistes prennent des mesures de rigueur budgétaire et s'alignent sur le tournant néolibéral pris par l'ensemble des pays européen ${ }^{5}$. Dans le domaine des politiques migratoires, Georgina Dufoix, nommée Secrétaire d'État aux Travailleurs immigrés en mars 1983, dénonce l'immigration clandestine et limite les possibilités de regroupement familial. Le décalage entre ce contexte de durcissement politique et l'impression d'une ouverture des possibles s'explique par le secteur dans lequel évoluent les commissaires de l'exposition, celui de la politique culturelle. Le début des années 1980 marque l'intensification d'une politique de promotion des cultures immigrées entamée sous le 
mandat de Valéry Giscard d'Estaing. Cette période constitue par ailleurs, pour beaucoup d'entre eux, une étape cruciale dans leur carrière professionnelle. La commissaire de l'exposition Josée Chapelle en témoigne dans un mail de réponse à une demande d'entretien : " Je serais très heureuse de vous rencontrer et de vous parler de cette exposition qui a été un grand moment dans ma carrière professionnelle ${ }^{6}$. » Pour cette documentaliste de formation, qui n'a aucune expérience dans ce domaine, être nommée commissaire d'une exposition dans une institution mondiale de l'art contemporain constitue une opportunité inespérée. De même, Pierre Saragoussi - qui a joué un rôle majeur dans l'obtention de subventions publiques pour financer l'exposition en intervenant personnellement auprès du Premier ministre - vient d'être nommé membre de la Caisse des dépôts et consignations, une institution financière de premier plan alors qu'il se définit lui-même comme un "enfant de l'éducation populaire " " qui n'appartient pas aux grands corps de l'État. Pour Josée Chapelle et Pierre Saragoussi, promouvoir le travail des artistes d'origine immigrée obéit autant à un projet artistique qu'à un engagement moral visant à offrir aux enfants d'immigrée des opportunités dont ils ont eux-mêmes bénéficié. Ils croient en la possibilité de briser les hiérarchies sociales en soutenant personnellement les projets artistiques et parfois les projets de vie des enfants d'immigrés. Ainsi, quand une jeune fille décide, au terme de l'exposition, de quitter la région de Montbéliard où elle a grandi pour s'installer à Paris, elle est soutenue par Josée Chapelle et son adjointe : «Fatima venait d'avoir 18 ans [elle montre sa photo sur le catalogue de l'exposition]. Elle m'a annoncé qu'elle restait à Paris. Comme elle ne savait pas où habiter, je l'ai accueillie chez moi durant trois mois après l'inauguration ${ }^{8}$ ". Quelques mois après l'exposition, Josée Chapelle participe également à Convergence 84, une traversée de la France à mobylette pour revendiquer la reconnaissance des enfants d'immigrés. Elle avait rencontré José Vieira et Farida Belghoul, les leaders de cette mobilisation, au cours de l'exposition où ils avaient tous les deux présenté leur travail.

\section{Les ambiguïtés d'une reconnaissance}

Dès l'origine, le projet dévoile son ambiguïté : il se présente à la fois comme une exposition d'œuvres d'art et comme une exploration anthropologique de la vie des enfants d'immigrés dans les banlieues française. La plupart des textes de lépoque insistent sur l'idée que ce sont autant les artistes que leur travail qui est mis à l'honneur. Le catalogue de l'exposition assimile ainsi les artistes à des « sujets » de l'exposition : "Le catalogue souhaite rendre compte de la richesse de l'expression des jeunes issus de l'immigration qui sont à la fois sujets et acteurs de la manifestation'. " Cette confusion entre les acceptions artistique et anthropologique de la culture a des conséquences sur la réception critique de l'exposition. Un rapport rédigé à la demande du Centre Pompidou décrit ainsi cette exposition comme un moment où c'est autant le travail de l'artiste que l'artiste lui-même qui s'expose : «Au premier semestre 1984, des jeunes maghrébins, portugais, asiatiques, africains exposaient / s'exposaient au Centre Georges Pompidou ${ }^{10}$ ". Ces textes montrent que les exposants ne peuvent, à l'instar des autres artistes, disparaître derrière leur œuvre d'art qui n'existe ici qu'en relation avec un discours sur l'artiste "d'origine immigré ».

L'absence, parmi les exposants, de certains artistes dont le travail porte pourtant sur le thème de l'immigration montre d'ailleurs qu'il est nécessaire d'être soi-même « issu de l'immigration " pour pouvoir exposer. Le cas de Antoine de Barry - un artiste plasticien qui a monté une exposition à partir de cartes d'identité de travailleurs marocains - signale que le recrutement repose en partie sur un critère ethnique. Josée Chapelle justifie dans un entretien le refus d'exposer le travail de ce plasticien : « Quand il est venu me montrer son travail, nous avons discuté et 
je lui ai dit qu'il n'était pas dans le sujet parce qu'il traitait de la première génération et puis c'est un artiste plasticien français, pas du tout... [silence] ${ }^{11}$. » Cet extrait montre que ce n'est pas seulement le travail de l'artiste, mais aussi sa personne qui sont au fondement de la fin de non-recevoir qui lui est adressée. Si cet artiste a finalement pu présenter un travail, c'est parce qu'il a collaboré avec

La possibilité d'investir le

Centre Pompidou, de quitter

la région de Montbéliard

représente pour

ces femmes une opportunité

de reconnaissance sociale,

la possibilité d'intégrer un groupe de pairs et un réseau professionnel. des jeunes filles de Montbéliard, elles-mêmes « issues de l'immigration maghrébine ». Les hésitations et les silences qui ponctuent l'entretien au moment de nommer ce qui différencie le plasticien des autres exposants sont significatifs de la gêne que suscite un critère de sélection ethnique jamais nommé comme tel. Ce critère ethnique a pour conséquence de faire passer au second plan les considérations sur la qualité artistique des œuvres présentées. Un article publié dans la revue de l'association Inter Service Migrants, chargée de mettre en place la partie « spectacle vivant » de l'exposition, reconnaît que le critère esthétique n'a pas toujours été considéré comme essentiel dans la sélection des œuvres d'art: «Les [films] les plus intéressants cependant, même s'ils peuvent quelquefois être techniquement imparfaits, sont ceux qui ont été réalisés par les premiers concernés, c'est-à-dire les jeunes immigrés ${ }^{12}$. 》 Un autre article de cette même revue souligne les difficultés rencontrées par les membres de l'association pour trouver des œuvres d'art qui répondent à ce critère : «Pour organiser une semaine de cinéma à Beaubourg, nous avons eu quelques difficultés à répertorier des œuvres cinématographiques réunissant de réels critères esthétiques. Il n'existe pas un courant, une école de cinéma de jeunes immigrés, mais nous avons constaté que certains parmi eux avaient la trempe de cinéastes professionnels, même s'ils avaient suivi des voies détournées ${ }^{13}$.
Cette difficulté à mettre la main sur une introuvable " culture immigrée » amène cette association à tenter de la faire émerger en finançant des projets artistiques en lien avec l'exploration des mondes immigrés. Inter Service Migrant a ainsi financé le film Le départ du père de Farida Belghoul, une jeune étudiante en économie. Bien que la jeune étudiante n'ait, à l'origine, aucune formation dans le maniement des techniques audiovisuelles, son projet de documentaire-fiction sur l'histoire de son père algérien intéresse l'association qui lui fournit des techniciens et du matériel pour mener à bien ce projet. Le soutien de cette association est lui-même le résultat d'une politique de subvention décidée au sommet de l'État par des experts qui préconisent d'accompagner l'expression culturelle des immigrés pour favoriser leur insertion sociale ${ }^{14}$.

\section{Être un artiste « d'origine immigrée » : les exposants entre critique et dépendance}

Comment les artistes qui ont participé à cette exposition ont-ils réagi à ces ambiguïtés du processus de sélection? La réaction des artistes dépend étroitement de leur parcours biographique et de leur carrière dans le monde artistique. On peut distinguer deux types de trajectoire parmi les exposants. Le premier groupe d'exposants réunit des individus ou des collectifs auxquels les organisateurs proposent de réaliser, à l'occasion de l'exposition, des projets témoignant de leur vie quotidienne d'enfants d'immigrés dans les quartiers populaires. N'ayant suivi aucune formation artistique, ces personnes sont accompagnées, dans la réalisation de leur projet, par des artistes qui les aident à convertir sous forme d'œuvre d'art un témoignage sur leur condition de vie. Josée Chapelle distingue ainsi, parmi les exposants, les « vrais artistes » des enfants d'immigrés à qui on offre la possibilité d'être, pour la

11. Entretien avec Josée Chapelle, op. cit 12. Images, spectacles musiques du monde. Revue d'animations culturelles d'inter service migrants, avril-mai-juin 1984, $n^{\circ}$ 1. 13. Images, spectacles musiques du monde. Revue d'animations culturelles d'inter service migrants, aout-septembre-octobre 1984, $n^{\circ}$ 2. 14. Voir, à ce propos, le rapport rédigé par Françoise Gaspard qui a guidé la politique culturelle en direction des populations immigrés « L’information et l'expression culturelle des communautés immigrées en France : bilan et propositions ", rapport remis au Ministère de la solidarité nationale, Paris, 1982. 
durée de l'exposition, des artistes : "On s'est trouvé comme ça avec un certain nombre de soutiens de personnes qui étaient des gens à qui on a demandé de faire des choses. Après, il y a eu des plasticiens, vraiment des artistes, Rachid Khimoune qui était le sculpteur, Mohand Amara qui a fait cette sculpture ${ }^{15}$. »C'est le cas du projet « La rupture » conçu par un groupe de jeunes femmes d'une association en collaboration avec l'artiste plasticien Antoine de Barry: «Six jeunes filles de 18 à 24 ans se sont regroupées à l'initiative de l'une d'elles, Leïla, avec le désir, vague au départ, de créer un livre illustré pour raconter ensemble leur vie au quotidien. Le projet d'exposition est venu concrétiser définitivement cette recherche. Antoine de Barry a été chargé de la mise en forme de ce travail pour l'exposition Les enfants de l'immigration ${ }^{16}$. » Pour ce groupe de jeunes filles, cette exposition offre une opportunité de quitter la région de Montbéliard et de fréquenter pendant quelques mois les acteurs du monde de la culture parisienne. Mais la participation à l'exposition n'est qu'une parenthèse de courte durée dans leur vie. Le cas de Malika, évoqué plus haut, constitue une exception puisque la jeune fille fait le projet de s'installer à Paris. Elle ne dispose néanmoins d'aucun réseau ni moyens financiers et reste entièrement dépendante de l'aide matérielle que lui fournissent la commissaire et son adjointe : « Je l'ai revue il n'a pas longtemps, elle est mère de famille et a trois enfants. Si elle est retournée un peu à Montbéliard, elle est restée à Paris. Par la suite, Véronique l'a prise comme nounou pour ses enfants. ${ }^{17}$ ".

Pour un deuxième groupe d'exposants, qui ont suivi des trajectoires différentes, cette exposition présente des opportunités de carrières dans le monde artistique. Ce groupe rassemble des individus qui ont suivi une formation en art ou qui ont déjà réalisé des projets artistiques à Paris ou dans la région parisienne. C'est le cas, par exemple, de Mohand Amara et de Rachid Khimoune, deux artistes plasticiens diplômés des Beaux-Arts de Paris. Ces artistes entretiennent, à l'égard des organisateurs, un rap- port ambivalent qu'on pourrait qualifier de « dépendance critique ». Le sculpteur Rachid Khimoune témoigne ainsi de la déception qu'à entraîné sa prise de conscience des véritables raisons de sa présence à l'exposition : «J'aurais bien voulu être exposé au Centre Georges-Pompidou pour mon travail et non parce que je suis enfant d'immigré ${ }^{18}$. " Dans un entretien accordé aux organisateurs pour la préparation de l'exposition, la cinéaste Farida Belghoul témoignait du même type de frustration vis-à-vis de la difficulté à faire reconnaître la qualité artistique de ses films : "Nous, les enfants d'immigrés, sommes arrivés pour les raisons que vous connaissez à produire un certain nombre de choses et, visiblement, on ne nous accorde pas encore le statut d'auteur. À l'égard de mon film, beaucoup de gens pensent que c'est l'histoire de mon père, que c'est mon histoire à moi, que le personnage du père dans le film c'est forcément, comment dirais-je, la personnalité de mon père. Or mon père joue un rôle dans le film. Quant à moi, j’ai effectué un travail qui dépasse le cadre de ma famille $e^{19}$. " Alors que cette jeune femme a comme ambition d'être reconnue comme un auteur capable de transformer son expérience familiale en fiction documentaire, son film est reçu comme un reportage sur l'histoire de sa famille algérienne. Dans Cette exposition montre une tribune publiée dans qu'ilabien existé, une revue militante le jour danslafrance desannées de l'inauguration de l'exposi- 1980, contrairement tion, Farida Belghoul livre une à ce qu'avancent certaines vision critique de l'exposition : analyses, des politiques "Des ratons, jeunes de toutes les de «discrimination positives» couleurs, exposent à Beaubourg fondées sur des critères du 18 janvier au 23 avril $1984 . \quad$ "ethniques». La formule est plaisante. Exposer à Beaubourg, de nos jours, donne le frisson et un certain cachet. Tant mieux. Nous n'allons pas cracher dans la soupe ${ }^{20}$.» Le retournement du stigmate (" nous les ratons ») et l'interpellation sur la composition du panel d'exposant ("Ya-t-il un français dans l'expo ?») visent à rendre explicite un critère ethnique dont 
nous avons montré qu'il restait un non-dit dans le discours des commissaires de l'exposition. Néanmoins, cette tribune témoigne aussi des liens de dépendance entre Farida Belghoul et les représentants d'une institution culturelle qui lui a "donné sa chance ». L'expression "nous n'allons pas cracher dans la soupe » exprime le sentiment de dette qu'elle nourrit à l'égard des personnes qui lui ont offert l'opportunité de faire voir son film sur une scène majeure de l'art contemporain. La dénonciation des ambiguïtés entretenue par les commissaires de l'exposition s'accompagne ainsi d'une difficulté à sortir d'une dépendance à leur égard en l'absence d'autres circuits de distribution de son travail.

Les doutes que nourrissent les artistes sur les véritables raisons de la promotion de leur travail sont confirmés et renforcés par la réception critique de l'exposition. Commentant le dossier que la revue Im'média consacre à l'exposition ${ }^{21}$, un journaliste du Monde s'insurge que des personnes puissent critiquer une exposition qui les met pourtant à l'honneur : "Décidément les Beurs sont de sacrés râleurs. Organisez pendant trois mois une exposition de neuf cents mètres carrés sur "les enfants de limmigration", artistes, chefs d'entreprise, photographe ou boxeurs. Tentez d'y associer de plain-pied ces groupes de jeunes immigrés qui se sont multipliés depuis la reconnaissance du droit d'association pour les étrangers le $1^{\text {er }}$ octobre 1981. Laissez-leur la parole à travers des animations ou débats, quitte à bousculer les habitudes d'une institution qui, de la culture, pouvait avoir une vue plus étriquée. Cela n'empêche pas de nombreux exposants de distribuer un brûlot le jour même de l'inauguration en présence de M. Jack Lang, ministre de la Culture et Mme Georgina Dufoix, secrétaire d'État à la Famille, à la Population et aux Immigrés22. " Le titre d'un rapport rédigé par un chargé d'études du Centre Pompidou reprend cette idée d'un honneur fait aux enfants d'immigrés : «Les enfants de l'immigration et les honneurs de la cimaise. Radiographie d'une exposition $^{23}$ ». En présentant cette exposition comme une opportunité offerte aux enfants d'immigrés, les observateurs contribuent à en faire les bénéficiaires passifs d'un honneur. Ils relativisent ainsi la qualité intrinsèque du travail des exposants au profit d'une focalisation sur leur statut d'artiste « d'origine immigrée ».

\section{Conclusion}

L'exposition Les enfants de l'immigration a marqué la plupart des acteurs qui y ont participé. Les liens d'amitié, d'entraide et de reconnaissance mutuelle qui se sont noués entre eux montrent que cette exposition a été, au-delà d'un projet artistique ponctuel, le lieu d'une rencontre entre les artistes « d'origine immigrée » et les représentants installés du monde de la culture. La reconstitution des choix effectués par les organisateurs au moment de sélectionner les exposants comporte néanmoins des ambiguïtés. Le critère ethnique apparaît comme central mais demeure passé sous silence, si bien que les critiques expriment des doutes sur ce qui, du talent artistique ou de l'origine immigrée des artistes, explique leur présence à l'exposition.

Les chercheurs n'ont pas suffisamment mis en évidence les grands effets de ces petits gestes sur les parcours biographiques des personnes qui en ont été perçues comme les bénéficiaires : en "donnant leur chance » à des individus sans rendre explicites les raisons de ces opportunités, ils ont contribué à faire émerger ce que nos proposons d'appeler une "micro politique du soupçon ». Ces soupçons concernent des individus qui font partie des groupes sociaux les plus susceptibles de subir des inégalités en raison de leur appartenance ethno-raciale. Au contraire, ils pèsent rarement sur ceux dont la présence dans les plus hautes sphères sociales ne fait pas problème, voire apparaît comme naturelle et allant de soi. Le rôle du capital social dans les phénomènes de reproduction sociale démontre pourtant combien les classes supérieures bénéficient également d'« honneurs » et de «faveurs » qui ne sont jamais nommés comme tels. 